\title{
Relative validity and reproducibility of an FFQ to determine nutrient intakes of New Zealand toddlers aged 12-24 months
}

\author{
Emily $\bigcirc$ Watson ${ }^{1}$, Anne-Louise M Heath ${ }^{1, *}$, Rachael W Taylor ${ }^{2}$, Virginia C Mills ${ }^{1}$, \\ Ashleigh C Barris ${ }^{1}$ and Paula ML Skidmore \\ 'Department of Human Nutrition, University of Otago, PO Box 56, Dunedin 9054, New Zealand: ${ }^{2}$ Department of \\ Medicine, University of Otago, Dunedin, New Zealand
}

Accepted 2 April 2014: Final revision received 9 November 2014: Accepted 4 February 2015: First published online 31 March 2015

\begin{abstract}
Objective: The study objective was to determine the relative validity and reproducibility of a modified FFQ for ranking the nutrient intakes of New Zealand toddlers aged 12-24 months.

Design: Cross-sectional study.

Setting: Dunedin, New Zealand.

Subjects: One hundred and fifty-two participants completed a ninety-five-item FFQ twice, and five days of weighed diet recording (WDR), over one month. Validity and reproducibility were assessed for crude data and for data that were weighted for total fruit and vegetable intake (FV-adjusted).

Results: De-attenuated correlations between FV-adjusted FFQ data and WDR data ranged from $0.45(\mathrm{Zn})$ to $0.77(\mathrm{Ca})$. The percentage classified to the correct WDR quartile by the FV-adjusted FFQ data ranged from 34.6\% (total fat, $\mathrm{Zn}$ ) to $50.3 \%$ (Fe). Average gross misclassification was 3\%. Bland-Altman statistics showed crude data had a range of $128-178 \%$ agreement with the WDR and mean FVadjusted intakes had 112-160\% agreement. FV-adjusted intra-class correlations, assessing reproducibility, ranged from 0.65 (vitamin C) to 0.75 (Ca).

Conclusions: The Eating Assessment in Toddlers (EAT) FFQ showed acceptable to good relative validity, and good reproducibility, for ranking participants' nutrient intake and is able to identify toddlers at extremes of the nutrient intake distribution. It will be a useful tool for investigating toddlers' nutrient intakes in studies that require a method of dietary assessment with low respondent burden.
\end{abstract}

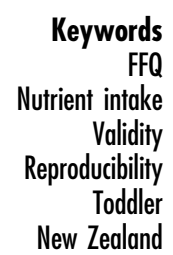

Dietary intake during the first 2 years of life is important not only because of the concerning rates of inadequate intake of some nutrients at this age ${ }^{(1)}$, but also because eating patterns developed as early as 9 months of age can continue throughout childhood ${ }^{(2)}$ and diet in infancy and toddlerhood may be associated with health indicators such as blood pressure several years later ${ }^{(3)}$. The measurement of dietary intake is, however, uniquely challenging in toddlers because of their high plate waste, the multiple carers often involved in their care (e.g. while in day care) ${ }^{(4)}$ and their rapidly changing dietary patterns as they are introduced to an increasing range of family foods. It is also important, particularly in a research setting, to minimise participant burden for busy families to maximise response rate and adherence.

FFQ assess usual dietary intake, have low respondent burden and are relatively inexpensive, but must be validated before use ${ }^{(5)}$. Six multi-nutrient FFQ have been validated in toddlers $^{(6-11)}$, but only three of these studies determined validity for toddlers separately from older children $^{(7,9,10)}$. The inclusion of older children may improve the apparent validity of an FFQ because an older child is more likely to be eating a meal similar to that of his/her family, including the person answering the $\mathrm{FFQ}^{(12,13)}$. Moreover, just one study ${ }^{(9)}$ had the recommended sample size of 100-200 participants ${ }^{(14)}$ and that study did not test the reproducibility of the FFQ.

The Prevention of Overweight in Infancy Study (POI.nz) ${ }^{(15)}$ is a randomised controlled trial in 800 families in Dunedin, New Zealand. An FFQ suitable for use with toddlers aged 12-24 months, with acceptable validity and reproducibility, was required for the POI.nz study so that nutrient intakes could be ranked while minimising respondent and researcher burden. No such FFQ was available.

The aim of the Eating Assessment in Toddlers (EAT) Study was, therefore, to determine the relative validity and reproducibility of a modified FFQ for ranking the nutrient intakes of toddlers aged 12-24 months. 


\section{Methods}

\section{Study design}

Participants attended two appointments four weeks apart. At the first, the FFQ, anthropometric measurements and sociodemographic questionnaire were completed. A five-day weighed diet record was completed over the following four weeks. The FFQ was then repeated at a second appointment, allowing both validity and reproducibility to be assessed. The nutrients of interest were energy, protein, total fat, carbohydrate, fibre, Ca, Fe, Zn, vitamin $\mathrm{B}_{12}$ and vitamin $\mathrm{C}$.

\section{Participants}

A convenience sample of 160 participants (primary caregiverchild pairs) was recruited from Dunedin, Mid Canterbury and Wellington (New Zealand) from September 2011 to April 2012. Primary caregivers were eligible if they were responsible for the care of a child 12-24 months of age, born at $\geq 36$ weeks' gestation, with no diagnosed illness known to affect growth or food consumption. The Human Ethics Committee of the University of Otago, Dunedin, New Zealand, granted ethical approval for the study and written informed consent was obtained from all primary caregivers.

\section{FFQ}

The FFQ was a modified version of the Southampton Women's Survey questionnaire designed to assess nutrient intake and dietary patterns in 12-month-old infants ${ }^{(7)}$. The food list was reconstructed to include foods consumed by at least $10 \%$ of New Zealand toddlers ${ }^{(16)}$. Products that were new to the market since the earlier study were identified in supermarket tours and added if they made a contribution to shelf space (indicating sales volume) similar to (or greater than) that of foods already on our list ( $11 \%$ of final food list). The FFQ has been validated for determining dietary patterns in New Zealand toddlers ${ }^{(17)}$.

Three novel components were added to the questionnaire: (i) cross-check questions for vegetable and fruit intake; (ii) use of child's palm volume to measure serving size; and (iii) collection of information on the amount offered as well as eaten. Cross-check questions asked for the overall frequency of vegetable (or fruit) consumption so that the frequency of consumption for each individual item within the vegetable (or fruit) section could be weighted to meet the overall frequency ('FV-adjustment') ${ }^{(18)}$. All portion estimates were modified from the household portion sizes (e.g. tablespoons) and food models used in the original FFQ: natural portion sizes (e.g. a banana) were used where possible and for the $58 \%$ of questions where natural portion sizes were not appropriate, parents were asked to describe portion size in terms of the child's palm volume (e.g. 'number of palms' of rice eaten; see 'Anthropometry' below). The modified questionnaire took into account 'plate waste' by asking for portion sizes of both the amount offered and the amount eaten.
The final FFQ was designed to be intervieweradministered and to rank toddlers 12-24 months of age by nutrient intake over the past four weeks. Ten frequency-response options were available, ranging from 'not offered this month' to an open-ended question for multiple times per day. The FFQ comprised ninety-five questions under eleven headings: (i) baby/toddler food; (ii) bread and crackers; (iii) breakfast cereals; (iv) rice and pasta; (v) meat, chicken, fish, eggs and beans; (vi) vegetables; (vii) fruit; (viii) dairy and dairy products; (ix) cakes, biscuits and snacks; (x) drinks; and (xi) other foods and drinks. Nutrient intakes were calculated using FOODfiles $2010^{(19)}$, except for toddler and baby foods (for which manufacturer and food label information was used). Where multiple foods were collapsed into one question, the foods were weighted using age-appropriate frequency and portion size consumption data ${ }^{(16)}$.

\section{Weighed diet record}

Five non-consecutive days of weighed diet records were collected over four weeks. The first day of recording was the day immediately following the participant's first appointment. The remaining four days were allocated so that in most cases a single day was collected each week, each participant collected at least one weekend day and all days of the week (including weekend days) were collected with approximately equal frequency across the total sample. Participants were given detailed verbal and written instructions at the first appointment and then contacted during the collection period so that they could ask further questions. Nutrient intake was analysed with the Kai-culator nutritional software package version 0.74 (Department of Human Nutrition, University of Otago, New Zealand) using the nutrient database FOODfiles $2010^{(19)}$, except for toddler and baby foods (for which manufacturer and food label information, or data from an earlier database (FOODfiles 2006) ${ }^{(20)}$, were used).

\section{Anthropometry}

Toddler length and weight were measured following standard protocols ${ }^{(21)}$. Length was measured using a Rollameter (Harlow Healthcare Rollameter 100, UK) to the nearest $0.1 \mathrm{~cm}$ (with duplicate measures within $0.7 \mathrm{~cm}$ ). Weight was measured using digital scales (Seca Alpha model 770; Seca, Hamburg, Germany) to the nearest $0.1 \mathrm{~kg}$ (with duplicate measures within $0 \cdot 1 \mathrm{~kg}$ ).

Palm volume was calculated as: palm thickness $\times$ length $\times$ width. Palm thickness was measured using an anthropometer (model 01291; Lafayette Instrument Company, Lafayette, IN, USA) to the nearest $0 \cdot 1 \mathrm{~cm}$ (with duplicate measures within $0 \cdot 1 \mathrm{~cm}$ ). The child's palm was scanned using a portable scanner (Canoscan LiDE 110; Cannon USA Inc., Lake Success, NY, USA) to determine length and width. The computer program Image (Free Software Foundation, Boston, MA, USA) was used to measure palm length (the distance from the middle of the 
wrist crease to the middle of the base of the third digit) and palm width (the maximum width across the metacarpalphalangeal joints II and V).

For all anthropometric measures, where duplicate measurements did not meet the specified criteria, a third measurement was made and the average of the two closest values (or median where the three values were equidistant) was used.

\section{Sociodemographic characteristics}

The NZDep2006 Index of Deprivation was calculated for all participants based on their home address (the NZDep2006 gives the least deprived $10 \%$ of New Zealand addresses a value of 1 , the $10 \%$ most deprived a value of 10$)^{(22)}$. Parents also completed a questionnaire on their child's age, sex, ethnicity and number of siblings.

\section{Statistical analysis}

All statistical analyses were undertaken using Stata/IC statistical software version $12 \cdot 0$ (2012). A $P$ value $<0.05$ was considered to indicate statistical significance.

The relative validity of the FFQ for assessing energy and nutrient intakes was calculated by randomly choosing the first or second FFQ for each participant and comparing this with his/her diet record data. The Shapiro-Wilks test and visual checks of histograms were used to determine the normality of the distributions for energy and the nutrients. Because the majority of the distributions were not normal, geometric means (and 95\% confidence intervals) were calculated for each nutrient for the FFQ and diet record. These means were compared using a paired $t$ test. Spearman's rank correlation coefficients were calculated comparing the FFQ and diet record data for each nutrient. Correlations of $0 \cdot 30-0 \cdot 49$ were considered

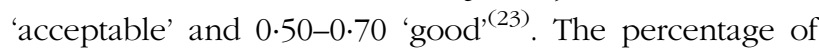
participants correctly classified and grossly misclassified into quartiles was calculated ${ }^{(5,23)}$. Correct classification was defined as the FFQ categorising the diet into the same quartile as the diet record. Gross misclassification was defined as the FFQ categorising the diet into the highest quartile when the diet record categorised it into the lowest, or vice versa. Bland-Altman analyses ${ }^{(24)}$ were used to assess the agreement between the two methods at the individual level.

The reproducibility of the FFQ was assessed by comparing the first and second administrations of the FFQ. Means were compared for each nutrient using a paired $t$ test and intra-class correlation coefficients were calculated to assess associations ${ }^{(5,23)}$

A second, identical, relative validity and reproducibility analysis was carried out using the vegetable and fruit cross-check questions to generate FV-adjusted data. A weighting factor was calculated (for the fruit and vegetable sections separately), as the total frequency with which the food group was eaten per day divided by the sum of all the individual frequencies in that section. Each individual item in the section was then adjusted by multiplying its individual frequency by the weighting factor.

De-attenuated validity correlation coefficients were also calculated. The FV-adjusted correlations were adjusted for the within-person variation occurring within the five days of diet records and within the two FFQ administrations ${ }^{(23)}$.

\section{Results}

One hundred and sixty parent-child pairs were recruited, of whom 152 completed both FFQ and the diet record, with one additional participant completing just the first FFQ and the diet record. This resulted in 153 participants (96\%) being included in the validity analysis and 152 (95\%) in the reproducibility analysis. The participants who withdrew from the study $(n 7)$ appeared to be more likely to have a female child, be of 'NZ European or other' ethnicity, have only one child and live in an area of low deprivation (NZDep2006 deciles 1-3), although this was not statistically tested because of the small number of participants who withdrew. The 153 children (51.3\% male) who had a completed FFQ and diet record had an average age of 16.8 months, weight of $11.0 \mathrm{~kg}$ and length of $81.5 \mathrm{~cm}$. The majority of the participants were New Zealand European (90\%) and had only one child ( $56 \%$ ). Only $11 \%$ of participants were from the three most deprived deciles of the NZDep2006 Index of Deprivation (compared with the expected $30 \%$ ).

\section{Relative validity}

The crude FFQ data gave significantly higher estimates of energy and nutrient intakes than the diet record for all nutrients $(P<0 \cdot 05$; Table 1$)$. Similar results were obtained after FV-adjustment with the exception of vitamin $\mathrm{C}$, which was not significantly different from the diet record (FV-adjusted $P=0.096$ ), although all FV-adjusted values (except vitamin $\mathrm{B}_{12}$ ) were lower and therefore closer to the diet record mean.

The average crude correlation between the FFQ and diet record was 0.50 ('good'), with a range from 0.37 ('acceptable') for $\mathrm{Zn}$ and total fat to 0.66 ('good') for $\mathrm{Ca}$ (Table 2). The correlations generally increased, albeit by a small amount, after FV-adjustment. The mean FV-adjusted correlation was 0.52 ('good'; ranged from 0.36 for $\mathrm{Zn}$ ('acceptable') to 0.68 for $\mathrm{Ca}$ ('good')). The correlations increased further after de-attenuation, to a mean of 0.62 ('good'; ranged from 0.45 for Zn ('acceptable') to 0.77 for $\mathrm{Ca}(>$ 'good')).

The percentage correctly classified into quartiles by the FFQ and diet record ranged from 30.1\% (total fat) to $48.4 \%$ (carbohydrate) for crude data (mean $40.1 \%$; Table 3). FV-adjustment did not improve classification (ranged from $34.6 \%$ for total fat to $50.3 \%$ for Fe; mean $40 \cdot 1 \%)$. The average percentage gross misclassification was similar for both crude data and FV-adjusted data, with 
Table 1 Average daily intake of selected nutrients from the diet record and FFQ among toddlers aged 12-24 months ( $n$ 153), Dunedin, New Zealand, 2011-2012

\begin{tabular}{|c|c|c|c|c|c|c|}
\hline \multirow[b]{3}{*}{ Nutrient } & \multicolumn{4}{|c|}{ Crude } & \multirow{2}{*}{\multicolumn{2}{|c|}{$\frac{\text { FV-adjusted } \ddagger}{F F Q}$}} \\
\hline & \multicolumn{2}{|c|}{ Diet record } & \multicolumn{2}{|c|}{$\mathrm{FFQ}$} & & \\
\hline & Mean* & $95 \% \mathrm{Cl}$ & Mean*, $†$ & $95 \% \mathrm{Cl}$ & Mean & $95 \% \mathrm{Cl}$ \\
\hline Energy (kJ) & 3494 & 3347,3647 & 5251 & 4994,5521 & 4646 & 4421,508 \\
\hline Protein $(\mathrm{g})$ & 33 & 32,35 & 54 & 51,57 & 51 & 49,54 \\
\hline Total fat $(\mathrm{g})$ & 28 & 27,30 & 49 & 46,52 & 45 & 43,48 \\
\hline Carbohydrate (g) & 111 & 107,116 & 149 & 141,157 & 124 & 118,131 \\
\hline Fibre $(\mathrm{g})$ & 7.9 & $7.5,8.4$ & $13 \cdot 4$ & $12 \cdot 6,14 \cdot 3$ & $9 \cdot 6$ & $9 \cdot 0,10 \cdot 2$ \\
\hline $\mathrm{Ca}(\mathrm{mg})$ & 556 & 511,605 & 820 & 758,888 & 782 & 720,850 \\
\hline $\mathrm{Fe}(\mathrm{mg})$ & $5 \cdot 8$ & $5 \cdot 4,6 \cdot 2$ & 7.4 & $6.9,7.9$ & 6.5 & $6 \cdot 0,6 \cdot 9$ \\
\hline $\mathrm{Zn}(\mathrm{mg})$ & $4 \cdot 2$ & $4 \cdot 0,4 \cdot 4$ & $7 \cdot 2$ & $6 \cdot 8,7 \cdot 6$ & $6 \cdot 7$ & $6 \cdot 4,7 \cdot 1$ \\
\hline Vitamin $B_{12}(\mu \mathrm{g})$ & 1.5 & $1.4,1.7$ & 2.4 & $2 \cdot 3,2 \cdot 6$ & 2.4 & $2 \cdot 3,2 \cdot 6$ \\
\hline Vitamin C (mg) & 47.5 & $42 \cdot 8,52 \cdot 8$ & $75 \cdot 4$ & $69 \cdot 1,82 \cdot 4$ & 36.6 & $40 \cdot 2,49 \cdot 0$ \\
\hline
\end{tabular}

*Geometric mean.

†One FFQ was randomly selected for each participant for the validity analysis.

F'FV-adjusted' is the crude data adjusted by the cross-check questions used in the fruit and vegetable sections of the FFQ.

Table 2 Correlations between the FFQ and diet record, and between the two FFQ, among toddlers aged 12-24 months ( $n$ 152), Dunedin, New Zealand, 2011-2012

\begin{tabular}{|c|c|c|c|c|c|c|c|}
\hline \multirow[b]{2}{*}{ Nutrient } & \multicolumn{4}{|c|}{ Relative validity* } & \multicolumn{3}{|c|}{ Reproducibility $\dagger$} \\
\hline & Crude & FV-adjusted $\ddagger$ & De-attenuated FV-adjusted§ & Previous studies\|l & Crude & FV-adjusted & D'Ambrosio $^{(11)}$ \\
\hline Energy (kJ) & 0.48 & 0.50 & 0.60 & $0.08-0.46$ & 0.72 & 0.72 & 0.63 \\
\hline Protein $(\mathrm{g})$ & 0.48 & 0.49 & 0.60 & $0.27-0.57$ & 0.71 & 0.70 & $>0.7$ \\
\hline Total fat (g) & 0.37 & 0.40 & 0.51 & $0.25-0.62$ & 0.70 & 0.70 & $>0.7$ \\
\hline Carbohydrate (g) & 0.58 & 0.59 & 0.73 & $0.25-0.52$ & 0.72 & 0.71 & $>0.7$ \\
\hline Fibre (g) & 0.59 & 0.56 & 0.69 & $0.23-0.38$ & 0.71 & 0.73 & $>0.7$ \\
\hline $\mathrm{Ca}(\mathrm{mg})$ & 0.66 & 0.68 & 0.77 & $0.26-0.74$ & 0.75 & 0.75 & $>0.7$ \\
\hline $\mathrm{Fe}(\mathrm{mg})$ & 0.61 & 0.63 & 0.75 & $0.31-0.48$ & 0.72 & 0.72 & $>0.7$ \\
\hline $\mathrm{Zn}(\mathrm{mg})$ & 0.37 & 0.36 & 0.45 & $0.30-0.62$ & 0.71 & 0.70 & - \\
\hline Vitamin $B_{12}(\mu \mathrm{g})$ & 0.41 & 0.39 & 0.49 & $0.24-0.47$ & 0.69 & 0.69 & $>0.7$ \\
\hline Vitamin $C(\mathrm{mg})$ & 0.48 & 0.57 & 0.72 & $0.19-0.58$ & 0.67 & 0.65 & 0.53 \\
\hline
\end{tabular}

*Spearman's correlation coefficients comparing the FFQ and diet record to assess validity.

tIntra-class correlation coefficients comparing the two administrations of the FFQ to assess reproducibility.

f'FV-adjusted' is the crude data adjusted by the cross-check questions used in the fruit and vegetable sections of the FFQ.

$\S$ 'De-attenuated FV-adjusted' is the FV-adjusted correlations adjusted for the within-person variation occurring between the five days of diet record and two administrations of the FFQ for each participant.

IIThe range of correlations from six multi-nutrient FFQ validation studies in toddlers $12-24$ months old ${ }^{(6-11)}$. Three studies reported Spearman's correlations $^{(7,9,10)}$, two studies reported Pearson correlations ${ }^{(6,11)}$ and one study reported energy-adjusted Pearson correlations ${ }^{(8)}$.

Table 3 Cross-classification by quartiles of nutrient intakes based on the FFQ and diet record among toddlers aged $12-24$ months ( $n$ 153), Dunedin, New Zealand, 2011-2012

\begin{tabular}{|c|c|c|c|c|}
\hline \multirow[b]{2}{*}{ Nutrient } & \multicolumn{2}{|c|}{ Crude } & \multicolumn{2}{|c|}{ FV-adjusted ${ }^{*}$} \\
\hline & $\%$ correctly classified $†$ & $\%$ grossly misclassified & $\%$ correctly classified $†$ & $\%$ grossly misclassified \\
\hline Energy (kJ) & 39.9 & $4 \cdot 6$ & 38.6 & $4 \cdot 6$ \\
\hline Protein $(\mathrm{g})$ & 37.9 & $3 \cdot 3$ & 38.6 & $2 \cdot 6$ \\
\hline Total fat (g) & $30 \cdot 1$ & 4.6 & 34.6 & 4.6 \\
\hline Carbohydrate (g) & 48.4 & 3.9 & $41 \cdot 6$ & 1.9 \\
\hline Fibre $(\mathrm{g})$ & $42 \cdot 7$ & 1.2 & $42 \cdot 5$ & 1.3 \\
\hline $\mathrm{Ca}(\mathrm{mg})$ & $45 \cdot 8$ & $2 \cdot 6$ & $46 \cdot 4$ & $2 \cdot 6$ \\
\hline $\mathrm{Fe}(\mathrm{mg})$ & $45 \cdot 8$ & $3 \cdot 3$ & $50 \cdot 3$ & $1 \cdot 3$ \\
\hline $\mathrm{Zn}(\mathrm{mg})$ & 34.6 & 4.6 & 34.6 & 3.9 \\
\hline Vitamin $B_{12}(\mu \mathrm{g})$ & $36 \cdot 6$ & 5.9 & $36 \cdot 6$ & 6.5 \\
\hline Vitamin C (mg) & 39.2 & 5.9 & $37 \cdot 3$ & 2.6 \\
\hline Mean & $40 \cdot 1$ & 4.0 & $40 \cdot 1$ & $3 \cdot 2$ \\
\hline
\end{tabular}

*'FV-adjusted' is the crude data adjusted by the cross-check questions used in the fruit and vegetable sections of the FFQ.

$\dagger \%$ correctly classified = percentage of children with a diet classified by the FFQ into the same quartile as the diet record. If the two methods were completely unrelated, $25 \%$ correct classification would be expected by chance.

$\ddagger \%$ grossly misclassified = percentage of children with diets classified by the FFQ into the highest quartile when the diet record classified them into the lowest quartile, and vice versa. If the two methods were completely unrelated, $12.5 \%$ gross misclassification would be expected. 
Table 4 Bland-Altman statistics comparing nutrient intakes from the FFQ and diet record* among toddlers aged $12-24$ months ( $n$ 152), Dunedin, New Zealand, 2011-2012

\begin{tabular}{|c|c|c|c|c|c|c|}
\hline \multirow[b]{2}{*}{ Nutrient } & \multicolumn{3}{|c|}{ Crude } & \multicolumn{3}{|c|}{ FV-adjusted $\dagger$} \\
\hline & Mean \% agreement & $95 \% \mathrm{Cl}$ & Limits of agreement (\%) & Mean \% agreement $\ddagger$ & $95 \% \mathrm{Cl}$ & Limits of agreement (\%) \\
\hline Energy (kJ) & 150 & 144,157 & $89-254$ & 133 & 128,138 & $81-219$ \\
\hline Protein (g) & 164 & 156,172 & 89-303 & 155 & 148,163 & 84-285 \\
\hline Total fat (g) & 172 & 164,182 & 88-337 & 159 & 151,168 & 83-308 \\
\hline Carbohydrate (g) & 134 & 128,140 & $79-227$ & 112 & 107,116 & $67-186$ \\
\hline Fibre (g) & 169 & 159,179 & $82-348$ & 121 & 114,128 & $59-248$ \\
\hline $\mathrm{Ca}(\mathrm{mg})$ & 148 & 140,155 & $79-227$ & 141 & 134,148 & $76-262$ \\
\hline $\mathrm{Fe}(\mathrm{mg})$ & 128 & 120,136 & $58-280$ & 112 & 106,119 & $52-242$ \\
\hline $\mathrm{Zn}(\mathrm{mg})$ & 170 & 162,179 & $89-325$ & 160 & 152,168 & $83-306$ \\
\hline Vitamin $B_{12}(\mu g)$ & 159 & 148,171 & $65-388$ & 159 & 148,171 & $65-390$ \\
\hline Vitamin C (mg) & 159 & 144,175 & $46-551$ & 93 & 85,103 & $28-311$ \\
\hline
\end{tabular}

*Data from the diet record and FFQ were natural log-transformed, calculations performed, then answers back-transformed and multiplied by $100 \%$. †'FV-adjusted' was adjusted by the cross-check questions used in the fruit and vegetable sections of the FFQ.

$\ddagger$ Mean \% agreement $=\mathrm{FFQ} /$ diet record $(\%) ; 95 \% \mathrm{Cl}=95 \% \mathrm{Cl}$ of the mean \% agreement.

energy and all nutrients less than $7 \%$. Mean gross misclassification with $\mathrm{FV}$-adjustment was $3.2 \%$ compared with $4.0 \%$ for the crude data.

Crude mean percentage agreements between the FFQ and diet record were all greater than 100\% (ranged from $128 \%$ for $\mathrm{Fe}$ to $172 \%$ for total fat), showing the FFQ overestimated mean energy and nutrient intakes compared with the diet record (Table 4). Energy and all other nutrients, except vitamin C, were also overestimated by the FV-adjusted FFQ data (ranged from $112 \%$ for carbohydrate and $\mathrm{Fe}$ to $160 \%$ for $\mathrm{Zn}$ ) compared with the diet record. FV-adjusted vitamin $\mathrm{C}$ intake showed good agreement between the FFQ and diet record, as indicated by the mean percentage agreement (FV-adjusted: $93 \%$ (95\% CI 85, 103\%)). The limits of agreement were wide for energy and nutrients, whether or not they were FV-adjusted.

\section{Reproducibility}

There were no significant differences in mean energy or nutrient intakes between the first and second administration of the FFQ, except for FV-adjusted vitamin $\mathrm{C}$ intake (mean difference $=4.8 \mathrm{mg} ; P=0.02$; data not shown). The percentage difference between the first and second FFQ ranged from $0 \%$ (Zn) to $9.4 \%$ (vitamin C) for crude intakes and from $0 \%$ (fibre and $\mathrm{Zn}$ ) to $10.5 \%$ (vitamin C) for FV-adjusted intakes.

Table 2 reports the intra-class correlation coefficients used to assess reproducibility between the first and second FFQ. The intra-class correlation coefficients had the same mean $(0.71)$ and range $(0 \cdot 65-0.75)$ for crude and FV-adjusted data.

\section{Discussion}

The EAT FFQ was designed to rank the nutrient intakes of New Zealand toddlers aged 12-24 months. The FFQ showed acceptable to good validity, with even better reproducibility. However, as is typical of the FFQ method, it overestimated energy and nutrient intakes compared with the diet record and gave wide limits of agreement. It is therefore not an accurate measure of absolute intakes or appropriate for estimating the intake of individuals.

Correlations between the FFQ and diet record were higher for energy, carbohydrate, fibre and Fe than previously reported, and all other correlations lay within the range that has been previously reported in validation studies of FFQ used in toddlers ${ }^{(6-11)}$. The correlations improved after adjustment for total vegetable and fruit intake ('FV-adjusted' values). When the correlations were de-attenuated the correlations increased further, with all nutrient correlations, except those for total fat and $\mathrm{Zn}$, higher than previously reported and correlations for carbohydrate, $\mathrm{Ca}, \mathrm{Fe}$ and vitamin $\mathrm{C}$ exceeding 0.7 (i.e. above the range considered to reflect 'good' agreement).

The cross-classification results were similar to ${ }^{(10)}$, or better than ${ }^{(9)}$, those for the two other studies that have reported cross-classification. We found low rates of gross misclassification (all less than 7\%). These results, along with those from the correlation analysis, suggest that our questionnaire is a useful tool for identifying children with extremes of dietary intake and is an appropriate method for ranking toddlers according to energy and nutrient intakes.

FFQ commonly overestimate intake, with all six previous validation studies with toddlers reporting overestimation of all $^{(6,7,10)}$ or the majority ${ }^{(8,9,11)}$ of nutrients assessed. It has been suggested that portion size estimation is a significant contributor to the overestimation of intakes ${ }^{(6,10,11,25-27)}$. Certainly, toddlers eat small portions and tend to leave food uneaten. The present study took two unique approaches to address these issues. First, information was collected on the amount offered and then, separately, on the amount eaten to encourage parents to differentiate between the two. Second, the FFQ asked participants to describe most portion sizes in units of 'palm volume'. A child's palm is 
small and therefore possibly closer in size to the portions of food eaten than other measurements commonly used, such as cups. It is also present at all feeding occasions, in close proximity to the food being eaten. However, the FFQ still overestimated absolute energy and nutrient intakes by a significant amount, except for FV-adjusted vitamin C.

The reproducibility of the FFQ was consistently high with $\mathrm{FV}$-adjusted intra-class correlation coefficients ranging from 0.65 to 0.75 . In comparison, a review article of FFQ in all age groups found reproducibility correlations were usually between 0.5 and 0.7 for most nutrients ${ }^{(28)}$.

A challenge facing all FFQ validation studies is recruiting participants who are representative of the population in whom the FFQ will be used. Our study used a convenience sample of participants and it is likely that they were more motivated than the general public. There was some ethnic diversity in our study, however not at levels representative of the New Zealand population, and the study also over-represented participants from the lower and middle deciles of deprivation. Further investigation would be required if the FFQ was to be used in minority populations such as communities with high deprivation or those containing a high proportion of Māori or Pacific people. Another limitation was our exclusion of nutrient intake from breast milk. This was because the weighed diet record was not able to provide data on breast milk consumption, so could not be used to validate intake. Researchers would therefore need to use a validated method for estimating breast milk consumption alongside the FFQ if it was used in populations of breast-fed toddlers. Finally, the FFQ was administered to the primary caregiver only, even though toddlers in New Zealand often attend early childhood education centres for at least part of the day. However, parents were asked to report on foods eaten when the toddler was cared for by others when they knew what the child had eaten (for instance, when they had prepared food for toddlers to take with them) and to report the proportion of the child's food intake that they were able to describe, and we provided an additional diet record for other carers to use when the child was away from his/her primary caregiver.

A strength of the present study was its design, which allowed both validity and reproducibility to be assessed in parallel. This is important given that high validity does not necessarily result in good reproducibility. Another study strength was the large sample size (153 in the validity analysis). Validation studies should have a sample size between 100 and $200^{(14)}$, yet only two previous multinutrient FFQ validation studies that involved toddlers had at least 100 participants ${ }^{(8,9)}$. Also, three of the earlier studies used $24 \mathrm{~h}$ recalls as the reference method ${ }^{(6,8,11)}$. Our study was able to collect five weighed diet record days. The weighed diet record is the preferred method because it is not affected by the same errors, in particular memory lapses and portion size estimation, as the $\mathrm{FFQ}^{(5)}$.
Finally, we used cross-check questions for fruit and vegetable intakes and these improved the performance of the FFQ. The fruit and vegetable food groups have been shown to be particularly vulnerable to over-reporting in previous studies of $\mathrm{FFQ}^{(18)}$.

The EAT validation study scored $5 \cdot 0$ out of $7 \cdot 0$ (classified as 'good') using an unmodified version of the European Micronutrient Recommendations Aligned (EURRECA) Network of Excellence scoring system for assessing the quality of dietary intake validation studies ${ }^{(29)}$. The two missing points were for considering seasonality in the study design (this is not relevant for this FFQ, which is designed to assess the past four weeks of dietary intake because intake can change so quickly in this age group) and supplement use (which is minimal in toddlers; only $3 \%$ of the participants used supplements on a diet record day). Our study, therefore, met all relevant criteria for the EURRECA Network of Excellence scoring system.

\section{Conclusion}

The EAT FFQ has good relative validity when compared with a five-day weighed diet record and has high reproducibility when measured over one month. It is suitable for assessing absolute intake of $\mathrm{FV}$-adjusted vitamin $\mathrm{C}$, although not other nutrients. This is consistent with most other FFQ, which characteristically overestimate intake and do not perform well at the individual level ${ }^{(5)}$. The questionnaire is able to rank the diets of New Zealand toddlers and identify toddlers at extremes of the nutrient intake distribution, making it a useful tool for investigating toddlers' nutrient intakes in studies that require a method of dietary assessment with low respondent burden.

\section{Acknowledgements}

Financial support: E.O.W. and V.C.M. were in receipt of University of Otago Master's Scholarships at the time the data were collected. E.O.W. prepared the first draft of this paper while in receipt of a University of Otago Postgraduate Publishing Bursary. The funders had no role in the design, analysis or writing of this article. Conflict of interest: None. Authorship: A.-L.M.H. and R.W.T. designed the modified FFQ; A.-L.M.H., P.M.L.S. and R.W.T. designed the research; E.O.W. and V.C.M. conducted the research; E.O.W., V.C.M. and A.C.B. entered data; E.O.W. analysed data; E.O.W. wrote first draft of the manuscript; all authors had input into subsequent drafts; A.-L.M.H., P.M.L.S, and R.W.T. have primary responsibility for final content; all authors read and approved the final manuscript. Ethics of buman subject participation: The Human Ethics Committee of the University of Otago, Dunedin, New Zealand, granted ethical approval for the study and written informed consent was obtained from all primary caregivers. 


\section{References}

1. Soh P, Ferguson EL, McKenzie JE et al. (2002) Dietary intakes of 6-24-month-old urban South Island New Zealand children in relation to biochemical iron status. Public Health Nutr 5, 339-346.

2. Lioret S, McNaughton SA, Spence AC et al. (2013) Tracking of dietary intakes in early childhood: the Melbourne InFANT Program. Eur J Clin Nutr 67, 275-281.

3. Brazionis L, Golley RK, Mittinty MN et al. (2013) Diet spanning infancy and toddlerhood is associated with child blood pressure at age 7.5 y. Am J Clin Nutr 97, 1375-1386.

4. Emmett P (2009) Workshop 2: The use of surrogate reporters in the assessment of dietary intake. Eur J Clin Nutr $\mathbf{6 3}$, Suppl. 1, S78-S79.

5. Gibson RS (2005) Principles of Nutritional Assessment, 2nd ed. New York: Oxford University Press.

6. Parrish LA, Marshall JA, Krebs NF et al. (2003) Validation of a food frequency questionnaire in preschool children. Epidemiology 14, 213-217.

7. Marriott LD, Inskip HM, Borland SE et al. (2009) What do babies eat? Evaluation of a food frequency questionnaire to assess the diets of infants aged 12 months. Public Health Nutr 12, 967-972.

8. Blum RE, Wei EK, Rockett HR et al. (1999) Validation of a food frequency questionnaire in Native American and Caucasian children 1 to 5 years of age. Matern Child Health J3, 167-172.

9. Andersen L, Lande B, Trygg K et al. (2004) Validation of a semi-quantitative food-frequency questionnaire used among 2-year-old Norwegian children. Public Health Nutr 7, 757-764.

10. Andersen LF, Lande B, Arsky GH et al. (2003) Validation of a semi-quantitative food-frequency questionnaire used among 12-month-old Norwegian infants. Eur J Clin Nutr $\mathbf{5 7}$, 881-888.

11. D'Ambrosio A, Tiessen A \& Simpson JR (2012) Development of a food frequency questionnaire for toddlers of LowGerman-speaking Mennonites from Mexico. Can J Diet Pract Res 73, 40-44.

12. Livingstone MB, Robson PJ \& Wallace JM (2004) Issues in dietary intake assessment of children and adolescents. $\mathrm{Br} \mathrm{J}$ Nutr 92, Suppl. 2, S213-S222.

13. Huybrechts I, De Bacquer D, Cox B et al. (2008) Variation in energy and nutrient intakes among pre-school children: implications for study design. Eur J Public Health $\mathbf{1 8}$ 509-516.

14. Cade J, Thompson R, Burley V et al. (2002) Development, validation and utilisation of food-frequency questionnaires a review. Public Health Nutr 5, 567-587.

15. Taylor BJ, Heath AL, Galland BC et al. (2011) Prevention of Overweight in Infancy (POI.nz) study: a randomised controlled trial of sleep, food and activity interventions for preventing overweight from birth. BMC Public Health 11, 942 .

16. Szymlek-Gay E, Ferguson E, Heath A-L et al. (2010) Quantities of foods consumed by 12- to 24-month-old New Zealand children. Nutr Diet 67, 244-250.

17. Mills VC, Skidmore PML, Watson EO et al. (2014) Relative validity and reproducibility of a food frequency questionnaire for identifying the dietary patterns of New Zealand toddlers. J Acad Nutr Diet (Epublication ahead of print version).

18. Calvert C, Cade J, Barrett JH et al. (1997) Using cross-check questions to address the problem of mis-reporting of specific food groups on food frequency questionnaires. UKWCS Steering Group. United Kingdom Women's Cohort Study Steering Group. Eur J Clin Nutr 51, 708-712.

19. New Zealand Institute for Crop \& Food Research (2010) FOODfiles: Data files of the New Zealand Food Composition Database. http://www.foodcomposition.co. nz/foodfiles (accessed March 2015).

20. New Zealand Institute for Crop \& Food Research (2006) FOODfiles: Data files of the New Zealand Food Composition Database. http://www.foodcomposition.co.nz/ foodfiles (accessed March 2015)

21. de Onis M, Onyango AW, Van den Broeck J et al. (2004) Measurement and standardization protocols for anthropometry used in the construction of a new international growth reference. Food Nutr Bull 25, 1 Suppl., S27-S36.

22. Salmond C, Crampton P \& Atkinson J (2007) NZDep2006 Index of Deprivation User's Manual. Wellington: Department of Public Health, University of Otago.

23. Willett W (1998) Nutritional Epidemiology, 2nd ed. New York: Oxford University Press.

24. Bland JM \& Altman DG (1999) Measuring agreement in method comparison studies. Stat Methods Med Res 8, $135-160$.

25. Fumagalli F, Pontes Monteiro J, Sartorelli DS et al. (2008) Validation of a food frequency questionnaire for assessing dietary nutrients in Brazilian children 5 to 10 years of age. Nutrition 24, 427-432.

26. Wilson AM \& Lewis RD (2004) Disagreement of energy and macronutrient intakes estimated from a food frequency questionnaire and 3-day diet record in girls 4 to 9 years of age. J Am Diet Assoc 104, 373-378.

27. Huybrechts I, De Bacquer D, Matthys C et al. (2006) Validity and reproducibility of a semi-quantitative food-frequency questionnaire for estimating calcium intake in Belgian preschool children. Br J Nutr 95, 802-816.

28. Cade JE, Burley VJ, Warm DL et al. (2004) Food-frequency questionnaires: a review of their design, validation and utilisation. Nutr Res Rev 17, 5-22.

29. Serra-Majem L, Frost Andersen L, Henríque-Sánchez P et al. (2009) Evaluating the quality of dietary intake validation studies. Br J Nutr 102, Suppl. 1, S3-S9. 